\title{
Design and Modeling of Nano-Robots Control in Medicine
}

\author{
Reza Soltani \\ Correspondence: Reza Soltani, Department of Mechatronic Engineering, Faculty of Mechanical Engineering, \\ University of Tehran, Tehran, Iran.
}

Received: January 10, 2018

doi:10.11114/set.v5i1.3406

\author{
Accepted: July 2, 2018 \\ Online Published: July 3, 2018 \\ URL: https://doi.org/10.11114/set.v5i1.3406
}

\begin{abstract}
This study aimed to present a new model to develop and expand nanotechnology in particular in the field of medicine. The subject under study focus on the control design of nano-robots for bio-molecular assembly manipulation, and use of evolutionary factors as a suitable method to gain the adaptive properties for proposed model is needed. Moreover, the study use of neural networks as the most practical method for the optimization problem of robot motion using a sensor based system. Thus, the study proposes a useful method within advanced graphics simulation for nano-assembly automation with its focus on an applied model for nano-medicine. Therefore, the study results should provide a great impact for effective design of control instrumentation, helping in the development of nanotechnology. The presented nano-robot model is required to survive and interact with a complex environment. Furthermore the nano-robot has to consider a pre-defined set of tasks both in a competitive scenario and in a collective environment. Nano-robot in a three-dimensional environment monitors organ inlets' nutritional levels, and assembling new biomolecules into that have to be delivered to the organ inlets with higher priority during each moment of our dynamic simulation. The nano-robot must avoid fuzzy obstacles, and must with proper time and manner react in real time for an environment requiring continuous control. In order to achieve the most pre-programmed set of behaviors the nano-robot uses a local perception through simulated sensors to effectively interact with the surrounding environment. The development of new concepts on nano-mechatronics and automation theory is focused on the problem of molecular machine systems. Finally a novel adaptive optimal method is described and the model validation through the application of nano-robot control design for nano-medicine confirmed.
\end{abstract}

Keywords: nano-robot control, nano-mechatronics, nano-medicine, bio-molecular

\section{Introduction}

Nanotechnology creates small, fast and inexpensive tools with new applications and in the medical field has revolutionized the treatment of diseases such as cancer, cardiovascular diseases, neurological disorders and other diseases. Developments of nanotechnology in various fields, especially in the field of sensors and computers lead to advent of nano- and micro-robots. Micro- and nano-bots on the one hand deal with large volumes of data and on the other hand, through sensors and actuators are associated with the physical world (Mohandesi and Nezhaf Koraim, 2007).

Nanotechnology can best be defined as a description of activities at the level of atoms and molecules that have applications in the real world. Nano-robots are the robots that are simply known as that controllable machine at the nano meter or molecular scale, composed of nano-components. More specifically, nano robotics refers to the still largely hypothetical nanotechnology engineering discipline of designing and building nano robots. Even though the field of nano robotics is fundamentally different from that of the macro robots due to the differences in scale and material, there are many similarities in design and control techniques that eventually could be projected and applied (Sivasankar and Durairaj, 2012).

Recently, field of nanotechnology and nano-engineering faced with new possibilities and challenges. The gap between the top down strategy and bottom-up strategy to build nanometer-sized electromechanical structures (NEMS) has been gradually reduced. Therefore, powerful tools to design and modeling nano-sized objects emerged. So, now we are entering a new stage to build NEMS based on nanotechnology (Mokhoff, 2003).

In the Europe, American and Japan research ministries have provided significant resources for the rapid development of nanotechnology, and in these fields spent million dollars to equip systems and tools with different types of nanotechnology (Scientific American, 2002). 
In the medical area, it is expected the same miniaturization of devices to have a direct impact on applied instrumentation and practices (Watson et al., 2009). Hence, the first class of nano-robots, that are expected to have effective applications in such areas as health care and environmental monitoring, are likely to emerge for the coming decades (Requicha, 2003).

The rise of nanorobots for medical diagnostics and treatments is arriving in the near future. In this study, a control method is designed so that the motion of a nanorobot can be controlled inside the blood vessel of the human body when we use them for medical therapies (Farahani and Farahani, 2016).

Two strategies top down and bottom-up for creating nano systems have been presented (Feynman, 1960). A combination of these two methods could be useful. Building first blocks through direct self-assemble to generate supra-molecules, and then convert them into more complex nano systems by smaller and smaller nano-manipulators. Hence, nano-manipulation, or positional control at the nanometer scale is a key technology for molecular nanotechnology (Drexler, 1981).

In the context of computer animation, motion control and planning can be used to effectively compute primary intentional motions. Examples include computing collision-free motions to accomplish high level navigation or object manipulation tasks (Kuffner, 1998; Bandi, 1998; Koga et al., 1994), or connecting different body configurations (Boulic et al., 1997; Ching and Badler, 1992). Motion planning is particularly suited to such tasks, since there is an infinite number of goal locations and obstacle in the environment.

Indeed path planning problems and motion control arise in different fields as robotics (Cavalcanti et al., 2007), assembly analysis (Cavalcant et al., 2009; Cavalcanti et al., 2008), virtual prototyping (Cavalcanti et al., 2007; Bar-On et al., 1993), and computer animation (Kuffner, 1998). Such problems involve searching the systems configuration space for a collision-free path and connecting start and goal configuration. Randomized algorithms for path planning and motion control successes in recent years and has better efficiency in handling problems with many degrees of freedom (Bar-On et al. 1993).

Jaiswal et al. (2013) studied the use of an artificial nano-medical erythrocyte which duplicates all of the important functions of the red blood cell by providing treatment for anaemia, heart attack, choking, lung diseases, asphyxia, and other respiratory problems. These nano-robots are able to keep a patient's tissues safely oxygenated for up to about 4 hours (at maximum dosage) if their heart has stopped beating in case of a heart attack.

Nanorobots are increasingly important in today's world, but there is still no good framework to design these kinds of robots, although in this field recently some studies have been carried out (Behkam and Sitti, 2006; Arora and Saini, 2013 and Farr et al. 2014).

Al-Arif et al. (2011) investigated the possibility of using Nanorobots in finding damaged nerve and delivering medicine to cure the demyelination. Due to the small size of these robots, the designed robots are very complicated and consist of very small components.

Boonrong and Kaewkamnerdpong (2011) proposed a swarm intelligence based control mechanism for swarm nanorobots that operate as artificial platelets to search for wounds. For both industrial and medical applications such as microsurgery, Pan et al. (2011) developed a prototype with a rotatable head and a body that has legs and fins.

Douglas et al. (2012) described a DNA nanobot shaped like a hexagonal tube, with its two halves connected by a latched hinge. When the little device recognizes a target cell based on its surface proteins, the two halves swing open like a clam to deliver a tiny but deadly cargo of drugs or nanoparticles.

\section{Proposed Model}

The aim of this work is to propose the analytical and computational method for a new control model, using computer aided design and real time physically based simulation, for the implementation of new concepts and methodologies that can support the automation of medical nano-robotics. The nano-robot model is pre-programmed to perform a set of tasks related to the nano-assembly automation and control. In the control model, ability of robotics features is considered important and complex issues to achieve molecular manipulation. For the effective development of nano technology can consider the implementation of new tools and systems adopting multidisciplinary methods. The demand for automatic manipulation, encoding and control of macro and nano structures of biological materials is paramount. In general, we can describe a nano-robotic molecular system as a system able to perform molecular manufacturing at the atomic scale; constituent robots are capable of interacting with the surrounding environment.

So, the main focus in this study is nano-robot control design for nano-medical application, while a set of pre-defined tasks is performed by nano-robots to deliver proteins in a 3D microscopic environment. In the next step, same biomolecules should be delivered to a set of organ inlets requiring drug or protein injection. For analytical analysis, we chose nano-manipulation in a liquid workspace, which is mostly relevant to biomedical applications. The proposed 
design has to be robust enough to perform in a complex environment with movement providing six-degrees-of-freedom. Taking into consideration all the characteristics described above, we adopted the use of non-deterministic approaches as the most feasible control method. Like some techniques inspired by biological and natural models, and evolving some capabilities characteristic of artificial life and intelligent factors, it should inherit some ideal counterparts for such a nano-robotic model.

Thus, a model using evolutionary techniques and artificial neural networks was adopted and finally the model as an adaptive model is used in the study. The reason for this choice is based on the fact that the nano-robot must be capable of reacting in real time, in accordance with different changes and requirements that come from the surrounding dynamic environment.

\section{Physical Simulation}

The physical simulation is a branch of computer graphics that has a great range of applications in different fields. Physical simulation for nanoCAD design, new devices at nano-scale in nanotechnology has great importance (Cavalcanti et al., 2008). With the application of virtual reality and dynamic collision detection many aspects for a better comprehension of kinetics features in the nanotechnology could be improved. The representation of diverse contacts in 3D are easily accompanied with the use of a virtual reality approach, where the exact conditions for dynamically contacted forces could be expressed. Although there are different methodologies for collision detection with bounding volumes, we have chosen the Interval Tree for 2D Intersection; this is the most suitable approach for the complexity of environments with different objects and robots moving all around the workspace, once its reduced number of pairwise checks improve the simulator performance. The computer graphics is providing a new opportunity for field of science include nanotechnology (Cavalcanti et al., 2009) moreover computer graphics will play a decisive role in the development of nanotechnology (Cavalcanti et al., 2008; Cavalcanti et al., 2006; Bar-On et al., 1993; Cavalcanti et al., 2007).

So despite the challenges and constraints, efficient and flexible algorithms to compute collision-free movement for certain set of work orders that are designed for a wide range of situations. The randomized path planner of Barraquand and Latombe (Barraquand et al., 1997) was an early attempt to solve problems with multi-dimensional configuration spaces. Theoretical results show that resolution of the geometry of the configuration space is a small roadmap can correctly capture the connectivity of the free space with a high probability (Hsu et al., 1997).

More precisely, incomplete roadmap represents the connectivity of the free space that decreases the number of sample points in the roadmap. The main issue affecting coverage of the free space is the presence of narrow passages in the configuration space (Hsu et al., 1998).

There are numerous variations on the basic roadmap strategy, most of which rely on different sampling techniques in an effort to reduce the computational costs. The roadmaps are especially suitable when multiple planning queries are given for a robot in the same static environment, since searching a roadmap is very fast (Amato and $\mathrm{Wu}, 1996)$. However, the overhead associated with roadmap is often too large for single-query planning problems in interactive environments (Boor et al., 1999). LaValle recently introduced the concept of Rapidly-exploring Random Trees (RRTs), for nonholonomic motion planning designed. RRTs have also been applied to kinodynamic planning problems in configuration spaces to 12 dimensions. For both holonomic and nonholonomic planning, the sampling technique show several desirable properties.

Further approaches were also proposed for example genetic algorithms, tabu search, neural networks that were applied to the problem of motion control (Cavalcanti et al., 2007). A mathematical description of the basic aspects related to the problem of motion control is detailed next.

The study of motion planning is a part of the control problem that has a great application for robotics control (Cavalcanti et al., 2008) many algorithms have been proposed dealing with motion planning optimization (Amato and Wu, 1996; Boor et al., 1999; Cavalcanti et al., 2007). Mobile robot requires a more elaborated motion algorithm, so robot can work in a more complex environment. Thus for such cases, the best way to deal with uncertain environments is to use non-deterministic approaches. Generally non-deterministic methods by a set of sensors will be supported robot when dealing with unpredictable situations.

\section{Description of Motion Control}

Basic motion planning can be characterized as an optimal control problem. In this section we are not using control theory to study issues such as robot dynamics and control, but simply redesign the basic motion planning problem.

Optimal control theory is a vast issue, and only some key definitions are provided here. Let $\mathrm{X} \subseteq \mathfrak{R} \mathrm{n}$ represent a state space in which $\mathrm{x} 0 \in \mathrm{X}$ represents the initial state of a system. Let $\mathrm{n} \mathrm{u}:[0, \mathrm{t} f] \rightarrow \Re$ represent a control function in which $[0, t \mathrm{f}]$ represents an interval of time. The control at time $t$ is given by $u(t)$, and the system state at time $t$ is given by $x(t)$. 
The system equation can be represented as $\mathrm{xf}(\mathrm{x}(\mathrm{t}), \mathrm{u}(\mathrm{t}))$, which defines how the state will evolve over time. A loss functional is defined and at any state path and control function as follows:

$$
\text { Equation 1: } \quad \mathrm{L}(\mathrm{x}(.), \mathrm{u}(.))=\int_{0}^{t f} \mathrm{l}(\mathrm{x}(\mathrm{t}), \mathrm{u}(\mathrm{t})) \mathrm{dt}+\mathrm{Q}\left(\mathrm{x}\left(\mathrm{t}_{\mathrm{f}}\right)\right)
$$

The integrand $1(x(t), u(t))$ represents an instantaneous cost, when integrated can be imagined as the total amount of energy that is expended. $\mathrm{Q}(\mathrm{x}(\mathrm{tf}))$ is a final cost that can be used to induce a preference over path that terminate in a particular portion of the state space by penalizing the final state of the system.

Also $\mathrm{t} f=\infty$ and describe an asymptotic final state $\mathrm{x}(\infty)$. Let the initial state, $\mathrm{x} 0$, is given. The optimal control design task is to select a control function $\mathrm{u}(\mathrm{x})$ that causes equation 1 minimized.

Considering that the basic motion planning could be expressed as a problem to compute a path $\tau[0,1] \rightarrow \mathrm{C}$ free so that $\tau(0)=$ qinit and $\tau(1)=$ qgoal, when such a path exists, there are natural choice for the state space as $\mathrm{X}=\mathrm{C}$ free.

Furthermore if robot dynamics were also included in the problem specification, then $\mathrm{X}$ might be expanded to include time derivatives on the configuration space. Next we define a simple system equation, $f(x(t), u(t))=u(t)$ for all $t$. This is not intended to be the most specific model of a particular robotic system, but rather it is used to encode the basic motion planning problem. We can assume for all $\mathrm{t}$ that the control input is either normalized, $\mathrm{u}(\mathrm{t})=1$, or $\mathrm{u}(\mathrm{t})=0$.

The initial state of the system is fixed, $\tau(0)=$ qinit . The loss functional can be simplified to $L(x(\cdot), u(\cdot))=Q(x(t f))$. We take $\mathrm{Q}(\mathrm{x}(\mathrm{tf}))=0$ if $\mathrm{x}(\mathrm{t} \mathrm{f})=$ qgoal, and $\mathrm{Q}(\mathrm{x}(\mathrm{tf}))=1$ otherwise. Thus the modeling partitioned the space of admissible controls into two classes: control functions that because the basic motion planning problem to be solved receiving zero loss; otherwise, unit loss is received.

The motion planning problem requires a collision-free path. This can be obtained by mapping the space of control functions into the space of state path for well-known obstacles. For $u(t), t>0$ and $x 0$, a state path $x u(t), t>0$ can be completely predicted. If $\mathrm{L}(\mathrm{xu}(\cdot), \mathrm{u}(\cdot))=0$, then the determined state path is a solution to the basic motion planning problem, which can be expressed as $\tau(\mathrm{s})=\mathrm{xu}(\mathrm{st} \mathrm{f})$.

The previous formulation considered all control inputs. By changing the functional loss, the optimal-path-length motion planning problem can be formulated:

Equation 2: $\quad L(X(),. U())=$.

otherwise $\rightarrow \infty$

The term $\int\|x\| d t \quad$ show the path length, and $x \&(T)=U(t)$ shown for all $t$.

\section{Sensor-Based Motion Control}

The role of perception for a given robot with predefined motor actions is to determine what actions take place and when. In this style of action-oriented perception, the action defines the perception what information is needed for the action to make its control decision. Thus perception for intelligent robots may be defined as "the process of gathering or receiving data about the environment and the agent itself"(Chenney, 1996). Architecture of intelligent agents usually provides method of interfacing the agent to the environment through sensors. Sensory information can be encoded at both a low level and a high level and utilized by high-level decision-making processes of the agent. We could summaries perception of the environment around a robot in two fundamental operations: gathering data about the environment, and interpreting the data. For a physical robot, gathering data involves devices such as cameras, laser rangefinders, and sonars, while interpreting data involves software algorithms, e.g. image segmentation, 3D model reconstruction, object recognition, motion estimation, etc.

Some research has been done on sensor-based motion, therefore perception is used to help decide what action the robot should take and when it should be performed, and such approach is also known in the literature as perceptual sensing.

\section{Artificial Neural Network}

The concept of neural networks and uses it return to human interest to simulate the brain (Boulic et al., 1997). Neural networks are computational models that are able to connect the relationship between inputs and outputs of physical 
systems by a network of nodes, determine where the activity of each of these connections by historical data set (learning process) finally, the model will be able to discover the laws of the inputs and outputs, but these laws are nonlinear and complex. A neural network consists of the components of layers and weight. Network behavior also depends on the relationship between members.

In general, three types of neurons in neural networks are:

1) Input layer: Get the raw data that is fed into the network.

2) Hidden layers: hidden performance by the input layer and weight with respect to the relationship between them and the hidden layer is determined. Weight between the input and hidden units determines when to activate a hidden unit.

3) output layer: output units performance due to hidden units and depending on activities based on the weight of the connection between the hidden and output units.

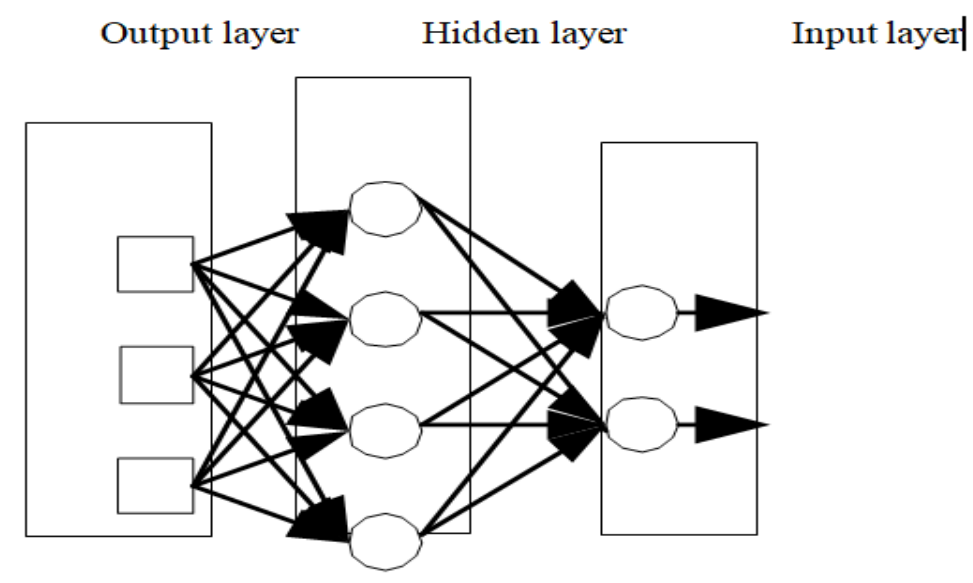

Figure 1. Simple artificial neural networks with a hidden layer

In order to design an artificial neural network is done the following steps:

According to the artificial neural network is based on data, data preparation is a critical step and the key to success in using neural networks. Principally using data as raw reduce speed and the accuracy of the neural network. In order to avoid such a case, as well as to equal the value of data in the network will be normalized (Soltani et al., 2010) a variety of methods are used to normalize the data is a common method as follows.

$$
\mathrm{y}=0.8 \times \frac{\mathrm{x}_{\mathrm{i}}-\mathrm{x}_{\min }}{\mathrm{x}_{\max }-\mathrm{x}_{\min }}+0.1
$$

Where $y$ is normal value of the parameter, $x_{i}$ the actual value of each parameter, $x_{\max }$ the highest value and $x_{\min }$ the lowest value is desired.

2) Select the three categories of training data, validation and test for a variety of models used:

In order to network correct training and prevent problems such as network saturation and weight meaningless, typically $20 \%$ of the data for validation, $10 \%$ for the test and $70 \%$ for training network used. But in some cases can be experimentally and by changing the ratio achieved the better results.

\section{Genetic Algorithms}

Genetic algorithms is based on study on intelligent animals natural reproduction that the results in the production process such as natural beings reproduction for participating and match it with the design of intelligent systems have been used. Genetic algorithms are computationally simple and a research and efficient search in different areas could to solve the problem.

This is a reasonable approach that crosses a set of sample groups (populations) given the scope of the problem, selected and maintained. For example, optimization of some of these populations using the theory of stochastic assessment so-called genetic operators manipulated and evaluated. Each iterations with accordance to the production of the first population in the population and the new generation was looking for the right solution and assessment practice based on good values and superior by comparing the new population that has been done to solve the previous groups. Using genetic operator's reproduction is done to make decent income.

Assessment of natural system of organism's reproduction, it follows that the beginning of life is based on the structural arrangement of chromosomes. Relationship between chromosome and combination structure function is a natural 
selection. Genetic algorithms design variables, or properties that are uniquely expressed in a regular series are collected. Each design variables or the same set of genes as a string variable with the concept of chromosomes in normal life matched. Genes are smallest components of chromosomes and chromosomes are representative of an individual in the population. In the world of numbers and calculations any number is a member of the population and the smallest elements that can make up a number, are category 0 and 1 (bits). Fields 0 and 1 are so found which represents the width of changes in the scope of problem-solving and assessment tools are optimized by function. Following the general trend genetic algorithm based on Figure 2 examined.

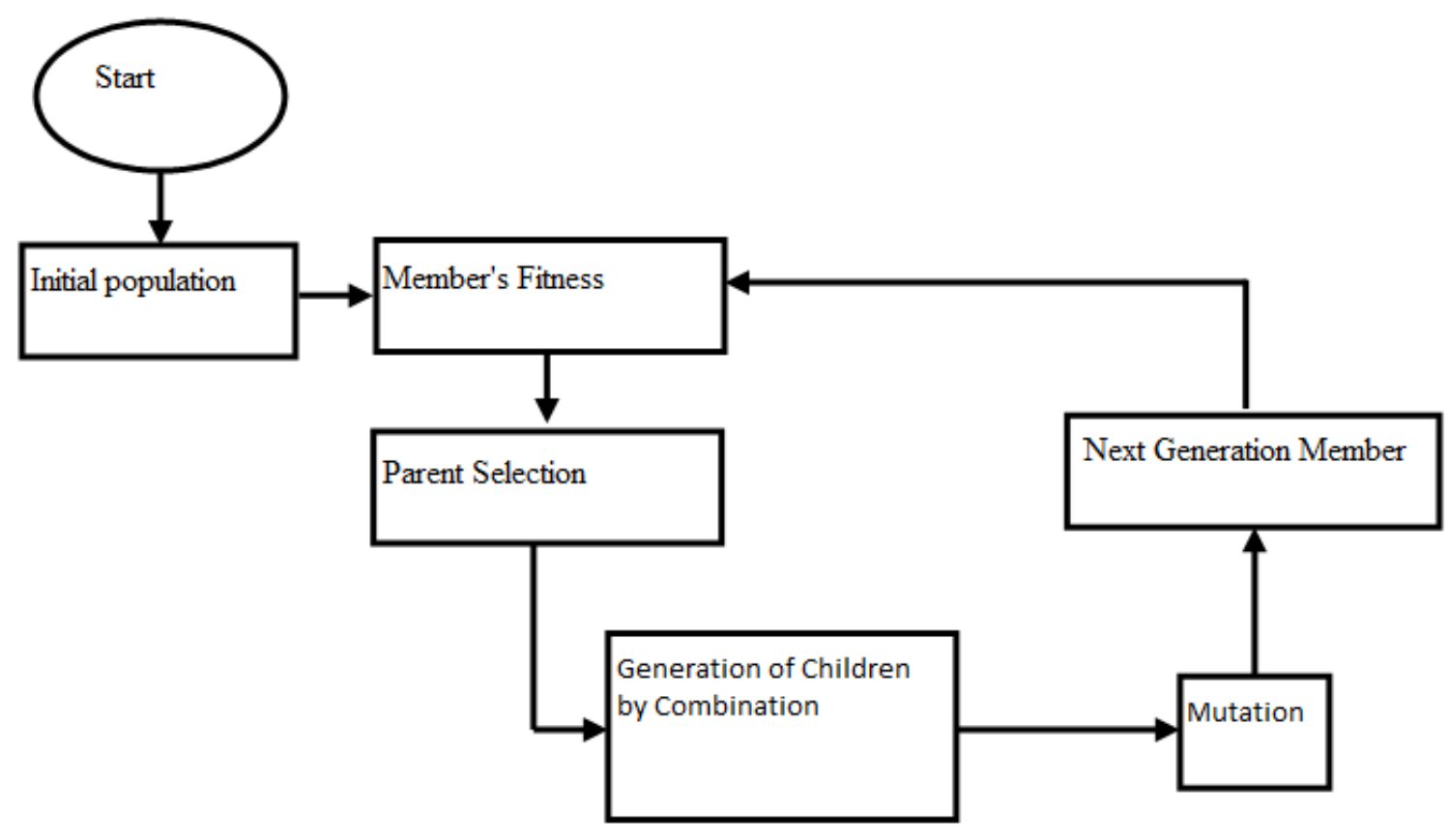

Figure 2. Overview of genetic algorithms

\section{Components of Genetic}

Genetic factors are entities which regulate the performance of the genetic algorithm and do it better and help account and are as follows: Number of population, rate of cross-operator and sudden mutation rate

\section{Population}

The size of population in the performance of the algorithm is effective. If we have a small population in a poor performance would not be an issue covered all possible space and looked at a large population is due to cover more space and the early resolution of convergence in the local limited and prevented.

While large population requires further evaluation in its production and consequently speed up the convergence rate slows.

\subsection{Rate of Cross-Operator (C)}

Rates of cross-operator are crucial component determining cross performance. In every generation a new population, the number of $\mathrm{C}^{*} \mathrm{~N}$ series consider changes in cross performance. Higher rate of cross operator means that the new fields will enter more quickly population if the rate of cross performance is too high, remove the strings faster than selection for recovery is done and conversely, the lack of this amount leads to a stasis that is responsible for reducing the rate of identification.

\subsection{Sudden Mutation Rate (M)}

Sudden mutation rate is likely to change something that the state of each bit of each discipline in a new population after each stage selected. The second stage is essentially a search function that increases the diversity in the population. Almost abruptly jumps to the value of $\mathrm{M} * \mathrm{~N} * \mathrm{~L}$ in any production $\mathrm{L}$ is the length of the string.

$\mathrm{M}$ avoid any situation that bit stay in a certain amount while the increase may be searching for something more effective.

Figure 3 shows the cycle of basic genetic algorithm 


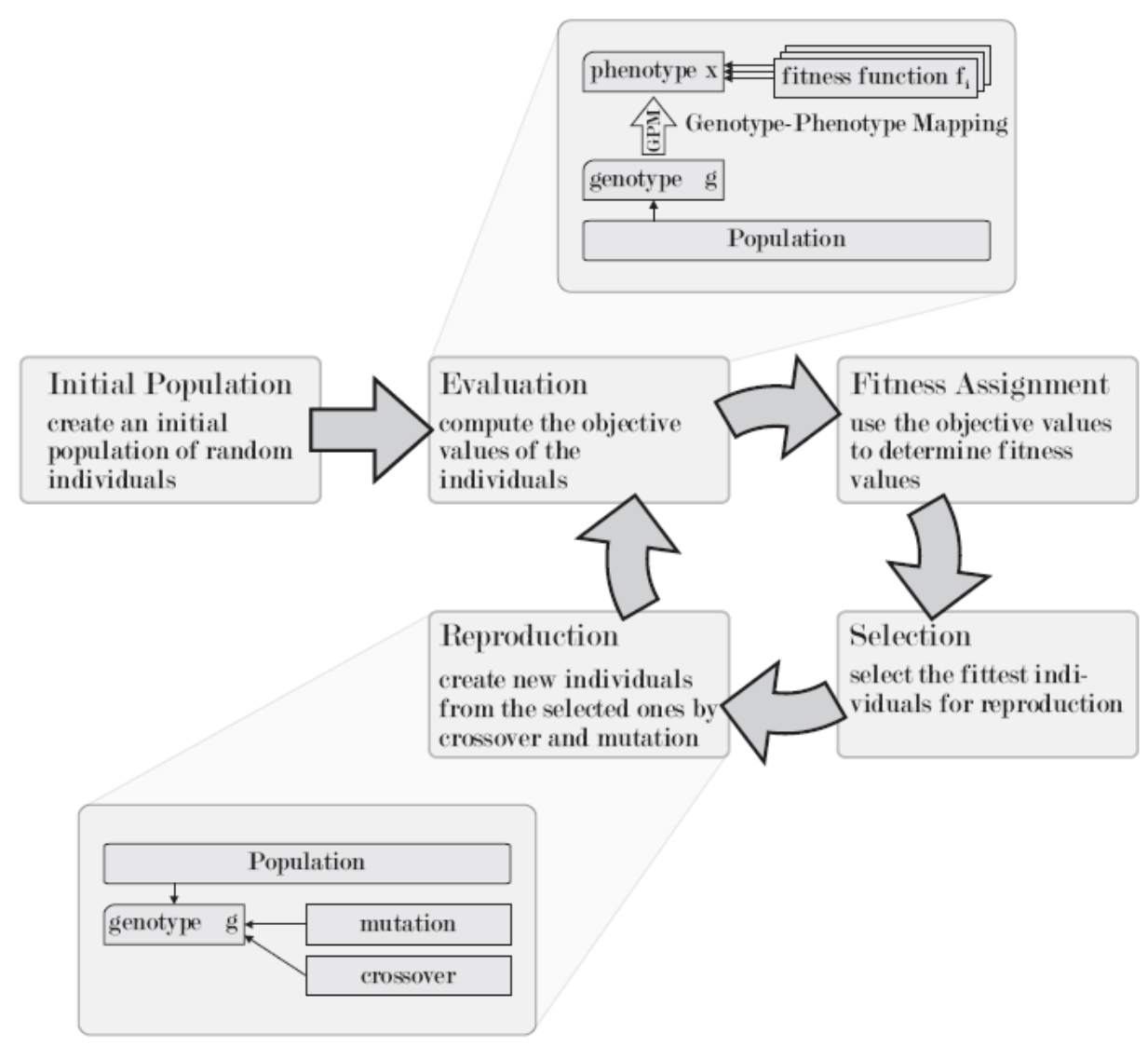

Figure 3. Basis cycle of genetic algorithms

\section{Parallel Processing for Robots Control}

In computer animation and physical simulation, can impose extreme loads on processing hardware. Motion dynamics and collision detection for complex environments on mobile robots need to interaction with each other and the objects surrounding them can be the most computationally intensive stage of the production process of an animation sequence.

Methods for decreasing the time spent on such activities must be a major priority if future requirements for complex, interactive scenes are met.

Distributed high-level robot control systems make use of functional parallelism by dividing the system into functionally different modules that run on different processing. A system with high efficiency can be achieved by concentrating on large and efficient software blocks with little inter-modular communication. 


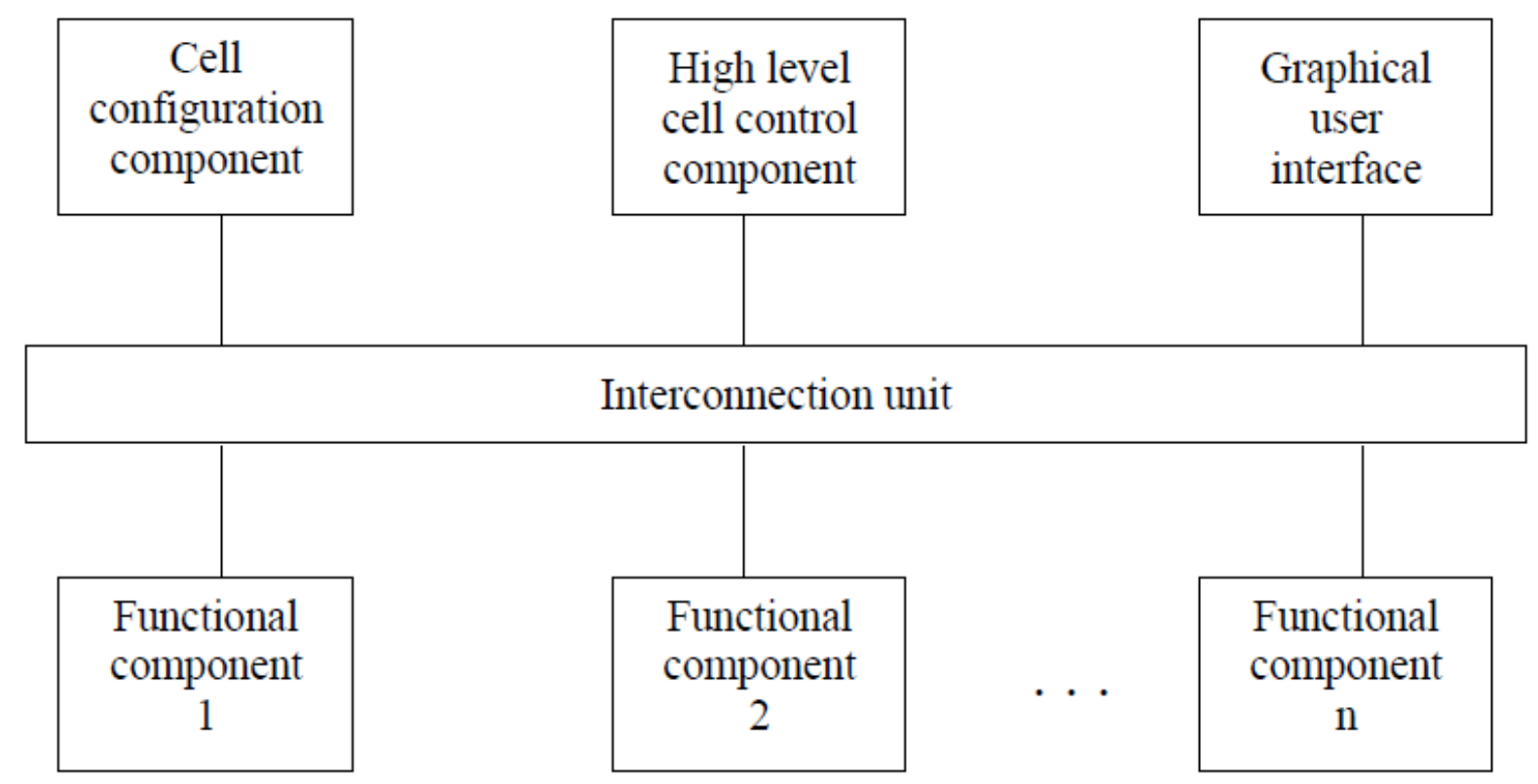

Figure 4. Concept of parallel robot control structure consisting of multiple components and an interconnection unit

\section{Parallel Architecture}

Before discussing parallel control architecture, it is important to explain what control architecture is. According to (Dilts et al., 1991), control architecture makes a control system from control components. The architecture determines the interrelationships between the components and the mechanisms for coordination. Requirements on robot control architecture can be described from a general point of view, for manufacturing systems, and for software architectures of robot control. Important requirements from the parallel processing point of view are required to attend flexibility. The goal of system concept provide scalable and flexible high level robot control architecture for a complex manipulation task in a 3D virtual environment with real time adaptive reaction. In order to provide high efficiency, our system is divided into subsystems with different functionality that may run on different processing and communicate by an efficient message passing protocol.

A subsystem is also called a component. A component implements a set of related functions and can either be a physical or a logical component. Logical components run as different processes in order to have the possibility to run them on different processing for higher efficiency. Additionally, a component process can be parallelized at the algorithm level like automatic motion and decision control.

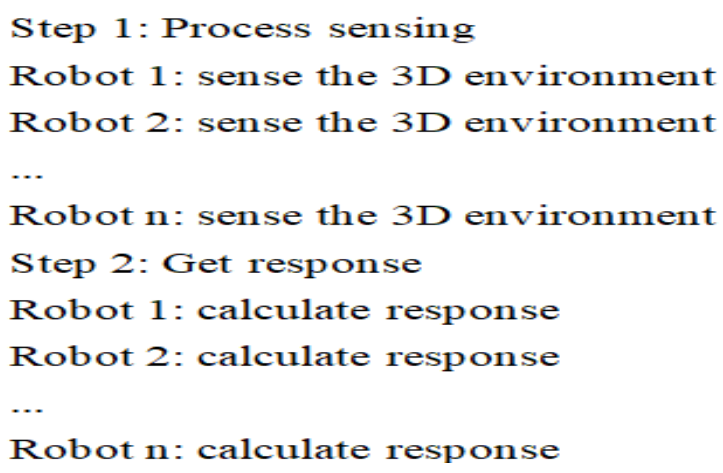

Figure 5. Processes of sensing and reacting in parallel with the environment

Processing demands could increase extremely rapidly for the physical simulation of mobile robots when using computer animation. Especially for the automation of robotics systems, which are characterized as systems composed of several functional modules, the use of methods for decreasing the operation time is more important. After identifying each functional module, the parallelization of such complex systems is an intensive field in computer science, even with an increasing level of processing power and memory capacity.

For our problem the same approach was used based on such concepts, considering the complexity of the scenery under study. 
Hence whole complex system divided into smaller functional parts, enables faster management and implementation, providing an architecture which is easier to test and to verify the robustness of each module. Afterwards such an approach seems to be a more suitable architecture for modeling robotics animation in computer graphics. To improve the simulator performance not only the systems were projected to run in parallel but also we have taken care to minimize as much as possible the intercommunication among the different modules to improve in the performance of the system.

\section{Proposed Control Design}

In this section distinct aspects of the main techniques required to achieve a successful nano-planning system design for a nano-robot model and testing the reliability of an adaptive behavior under diverse circumstances as a robust agent examined. It also shows the required architecture for a 3D visualization in real time. A new approach, using advanced graphics simulations for the problem of nano-assembly automation and its application in medicine with concepts derived from mobile robotics, is discussed.

Therefore study focus on dynamic control for nano-robot optimal performance as a suitable way to achieve a large range of tasks and biomolecular manipulation in a dynamic environment. In our described workspace representing a simplification of the human body, the nano-robot performs a pre-established set of tasks building nutrient molecules. Hence we discuss the main aspects in successful nano-robotics control modeling, proposing the main concepts required for development of molecular systems design.

\section{Virtual Environment}

Molecular machine systems could be described as a system capable of performing molecular manufacturing on an atomic scale. Regarding to nano-robotics control, computation is relatively cheap for macro-scale robotic actuators while arm motion is relatively cheap for nano-scale robotic actuators. So the computer control of arm path is the appropriate paradigm for macro-scale robots, but not for nano-scale robots. For nano-scale robots, the appropriate control is often path trial and error, also known as sensor based motion control (Cavalcanti et al., 2007).

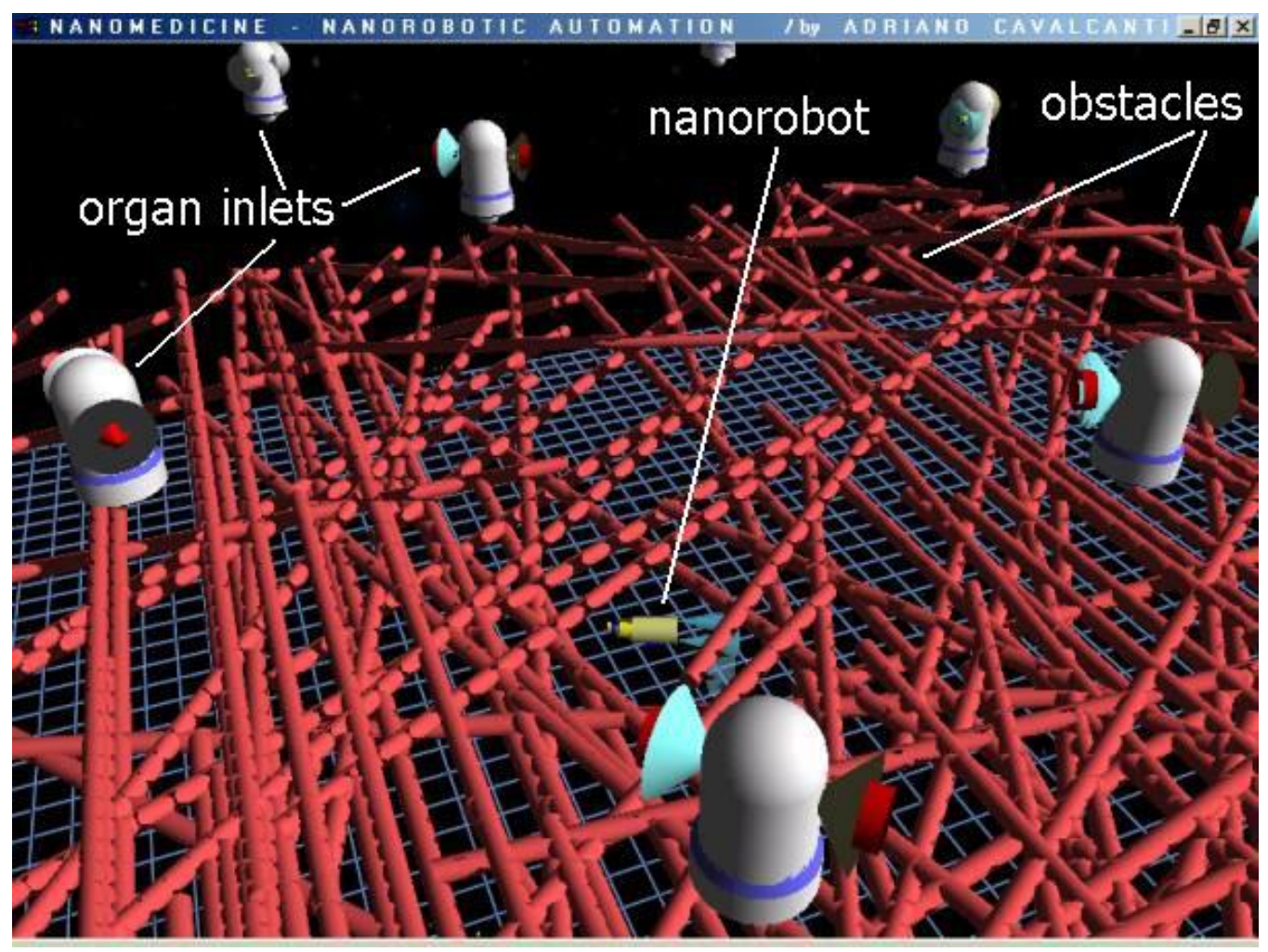

Figure 6. Top camera view in the virtual environment

Virtual reality was used for nano-robot design where the use of macro and micro-robotics concepts is considered as a practical approach and then we focused on the theoretical and practical aspects. Virtual environment in the study is inhabited by nano-robots, biomolecules, obstacles, and organ inlets. Each nano-robot measures $650 \mathrm{~nm}$ in length and $160 \mathrm{~nm}$ in diameter. The biomolecule has a diameter of $10 \mathrm{~nm}$ and each obstacle has a diameter of $120 \mathrm{~nm}$. The organ inlets are $400 \mathrm{~nm}$ in height and width with inlet orifices $720 \mathrm{~nm}$ in diameter. Top camera view can also see in virtual environment (Figure 6). 


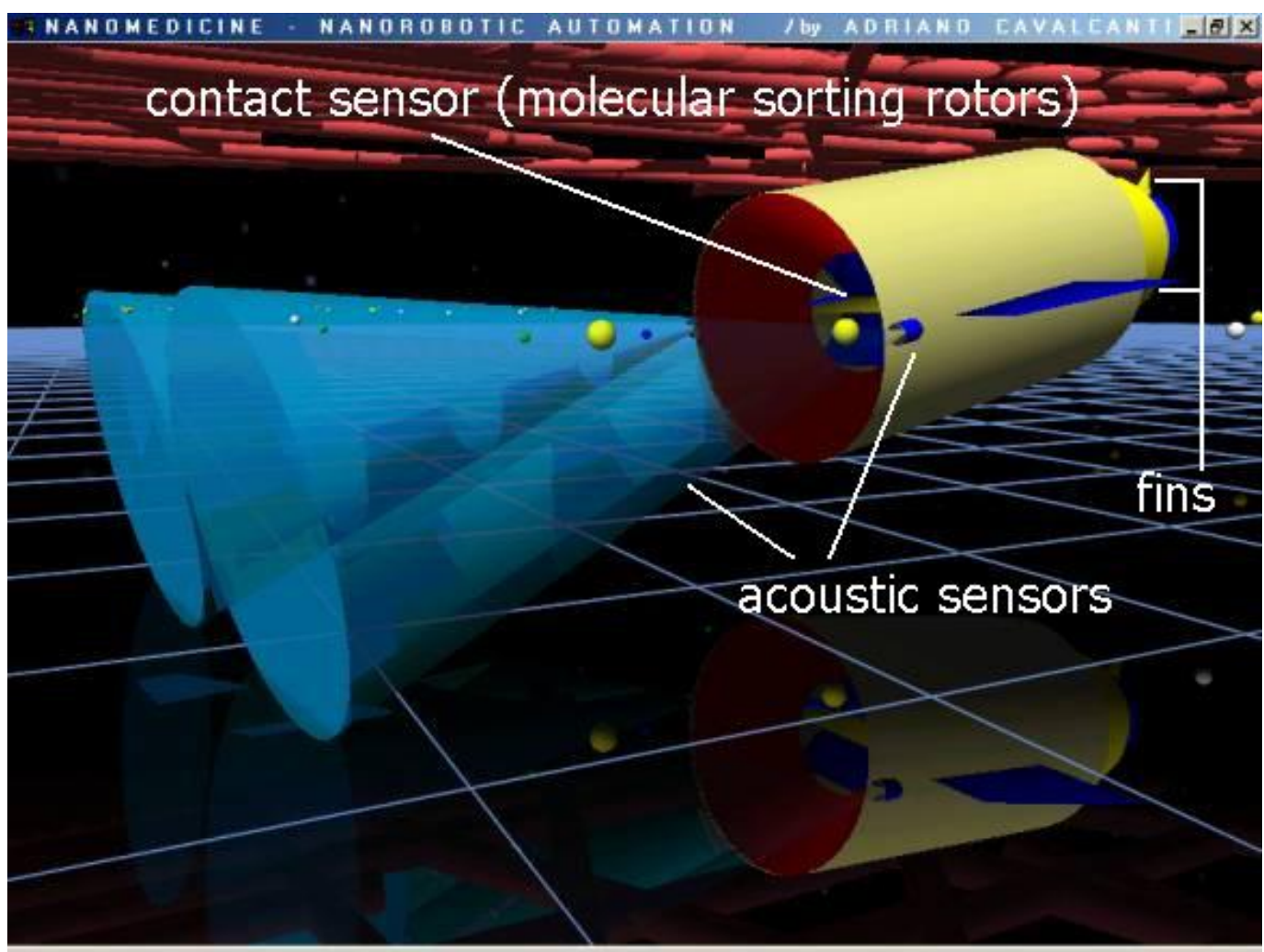

Figure 7. Molecular identification through collision contacts

Therefore a suitable starting point for hypotheses formulations and assembly system automation experiments was to consider the nano-robot design derived from biology and comprised of some basic nano-scale components such as molecular sorting rotors (Figure 6); to distinguish molecule types, molecular sorting rotor presents a series of chemotactic sensors.

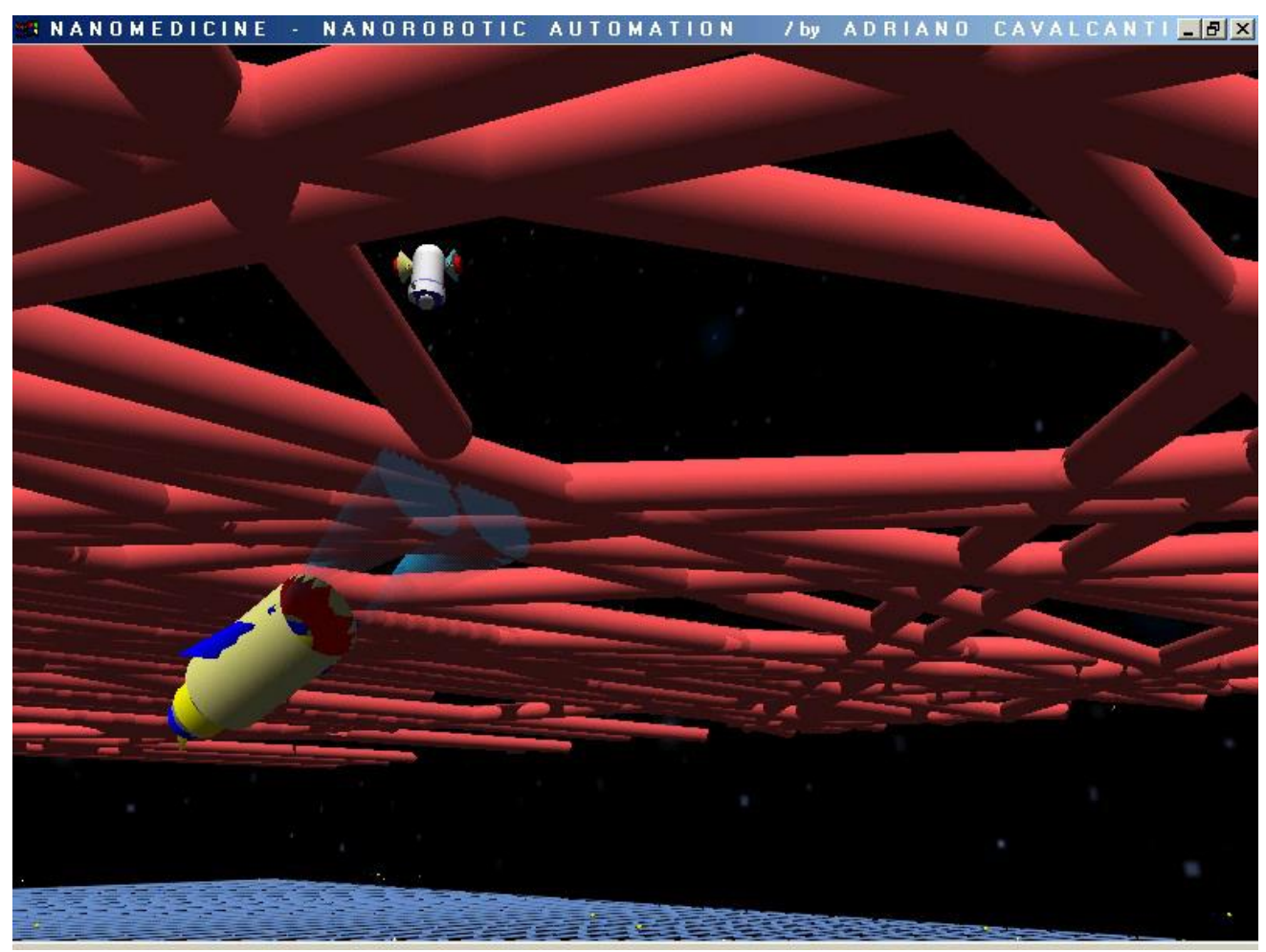

Figure 8. Robot avoid obstacle, sensing obstacles 


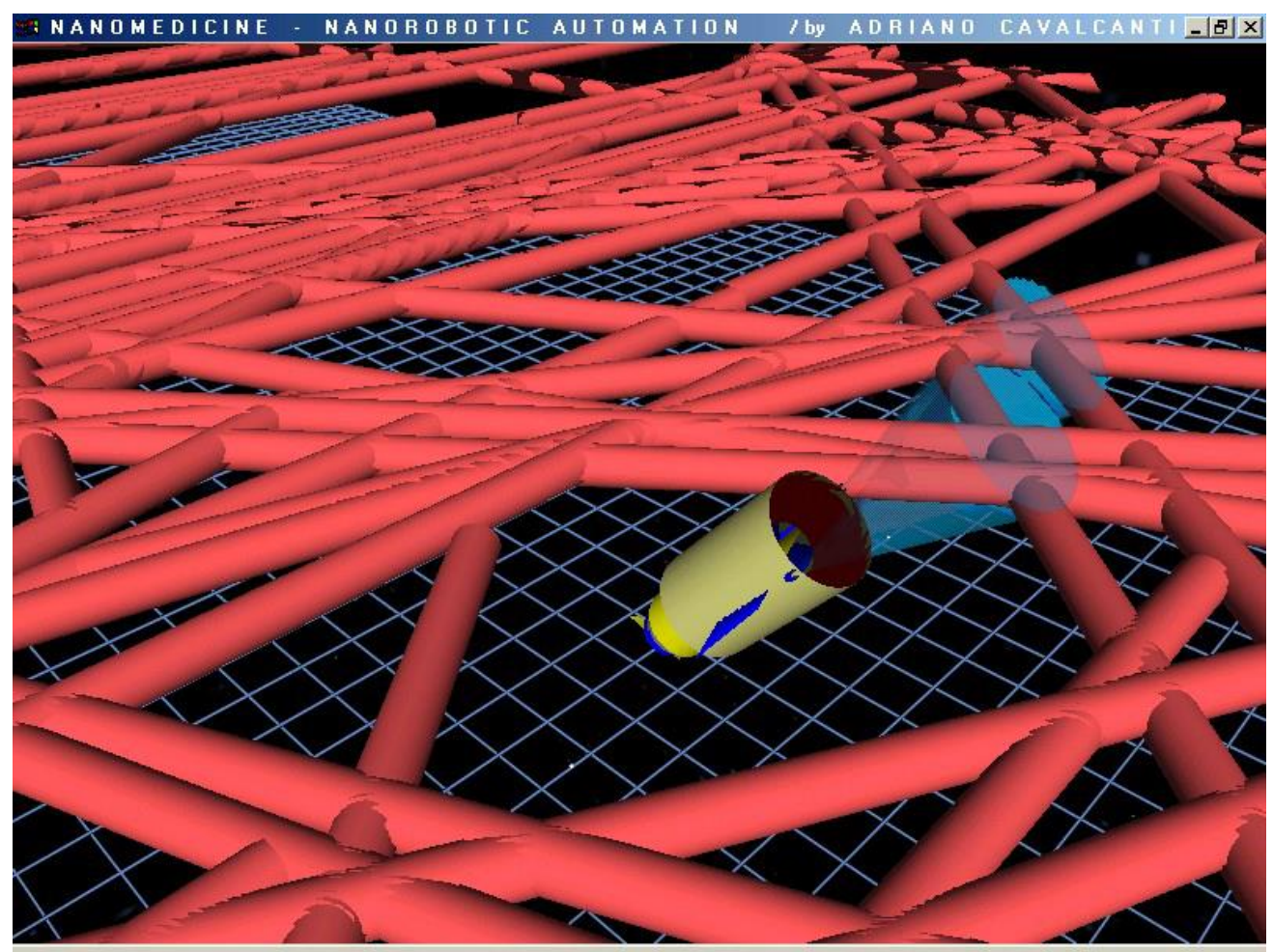

Figure 9. Robot avoid obstacle, finding path

Nano-actuator will be carried inside the nano-robot, and the nano-robot pushes the assembled molecule to the delivery point. The obstacles will be located in unknown probabilistic positions and the nano-robot has to avoid any collision with possible obstacles (Figures7 and 8).

Thus the delivery positions represent organ inlets and requiring proteins to be injected by the nano-robot in a well-known position, injection should be performed at time t. They will change their delivery orifice's colors and making it open or closed. Thus assembled molecules are delivered to specific locations by a nano-robot's at 2 micron 2 and embedded at appropriate spatial intervals across the organ inlets orifice, which will be open and be closed automatically within the nano-robot's delivery. The assembled molecule can be pumped by the molecular sorting rotors in 10 seconds.

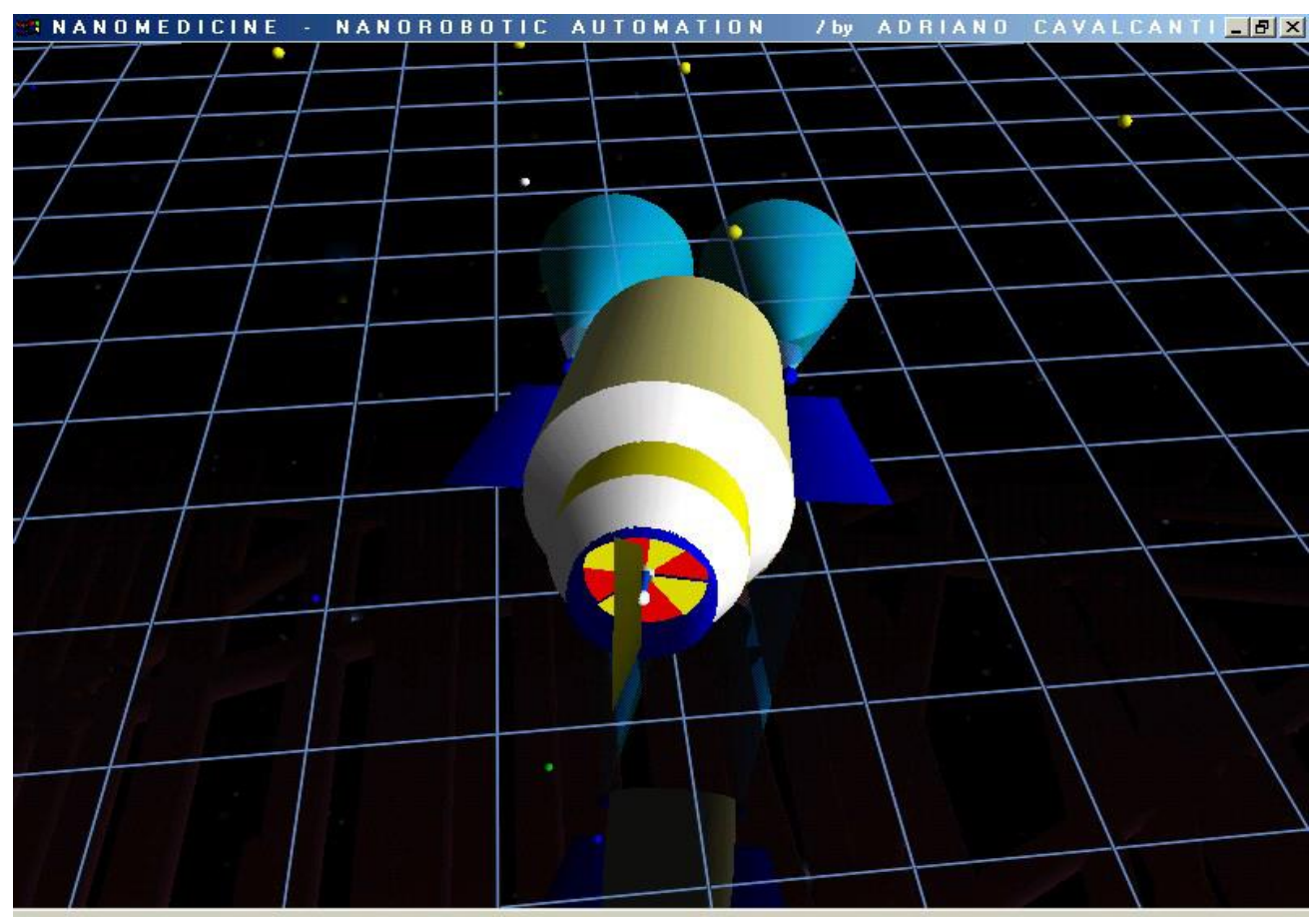

Figure 10. Nano-robots sensor, back view 


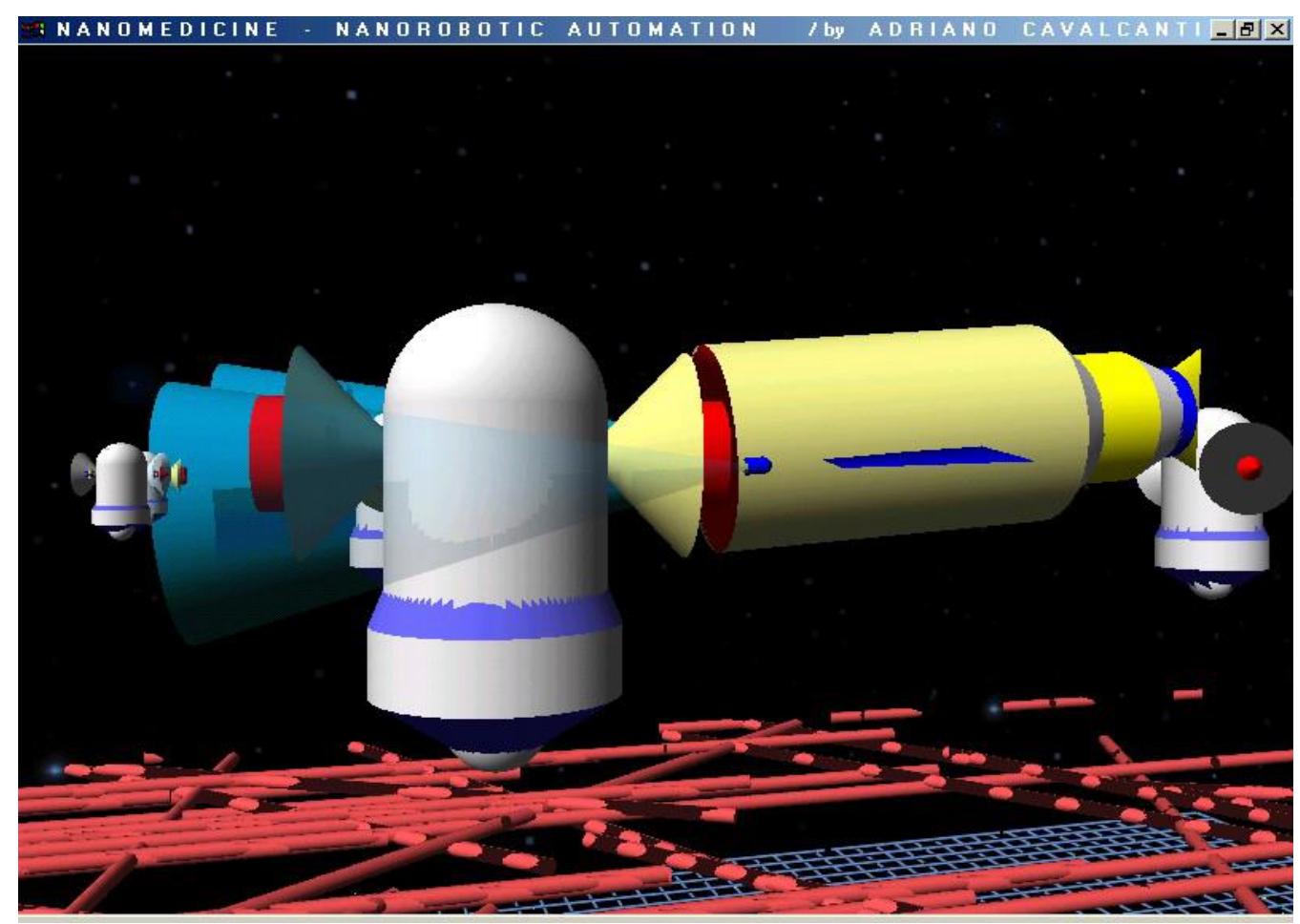

Figure 11. Nano-robot molecule delivery to the organ inlet

The development of a control model and the design of nano-robot automation have been described emphasizing the necessity to attain flexibility and robustness at the same time for any intelligent nano-molecular system prototyping, in relation to many aspects of the nano-worlds. The control model will be required to perform molecular manufacturing at nano-scale, which is even more evident for the specific problem that we are focusing on, that is related to the application of nano-robots in nano-medicine.

\section{Conclusion}

In the control model the most suitable method regarding the main aspects of adaptability, considering evolutionary and learning concepts, through the use of algorithms that are most applicable to complex problems, used. Moreover, the advantage of approach is to provide a modular behavior for the nano-robot, where the nano-robot is required to react in a well- way with the events that came up from the surrounding uncertain 3D environment. For such a goal the sensor-based concept, also known as position perception was used. The simulator implementation has required a higher performance.

The use of computer graphics for the design of the model has significantly helped understanding of many of those aspects related to nano-scale modeling. The main expected requisites for a representation of kinematics aspects at nano-scales were specified. Furthermore the follow-up and theoretical considerations, as well as numerical simulations and experimentation at nano-scale could be very hard, and even more so, to be designed and modeled, without the use of computer graphics.

Thus, the main prerequisite and techniques due to theoretical and practical characteristics and new paradigms for the development of control models applied and new field of nano-robotics created, and considered and discussed. We expect that the design approach presented here could serve as a framework for further studies on the field of nano-systems control design for mobile nano-robotics automation.

\section{References}

Al-Arif, S. M. M. R. M., Quader, N., Shaon, A. M., \& Islam, K. K. (2011). Sensor based autonomous medical nanorobots. A cure to demyelination. Journal of Selected Areas in Nanotechnology, 2(11), 1-7.

Amato, N., \& Wu, Y. (1996). A randomized roadmap method for path and manipulation planning. In Proc. of IEEE Int. Conf. Robotics and Automation, 113-120, Minneapolis, MN, 1996. https://doi.org/10.1109/ROBOT.1996.503582

Amir, F., \& Ali, F. (2016). An adaptive controller for motion control of nanorobots inside human blood vessels. Medical Communication. Biotech. Res. Comm., 9(3), 546-552.

Arora, R. K., \& Saini, R. P. (2013). Biosensors: Way of Diagnosis. International Journal of Pharmaceutical Sciences and Research, 4(7), 2517-2527. 
Bandi, S. (1998). Discrete Object Space Methods for Computer Animation. PhD thesis, Swiss Federal Institute of Technology, Lausanne, Switzerland, 1998.

Bar-On, D., Gershon, D., Israeli, A., \& Zuniga, G. (1993). TRACK II: A multi-processor robot controller. In Proc. CompEuro Int. Conf. on Computers in Design, Manufacturing, and Production, 86-93. https://doi.org/10.1109/CMPEUR.1993.289817

Barraquand, J., Kavraki, L. E., Latombe, J. C., Li, T. Y., Motwani, R., \& Raghavan, P. (1997). A random sampling scheme for path planning. In Int. J. of Robotics Research, 16(6), 759-774. https://doi.org/10.1177/027836499701600604

Behkam, B., \& Sitti, M. (2006). Towards Hybrid Swimming Micro-robots: Bacteria Assisted Propulsion of Polystyrene Beads. In: Proceedings of the 28th IEEE EMBS Annual International Conference, New York City, USA, 2421-2424.

Bohringer, K. F., Fearing, R. S., \& Goldberg, K. Y. (1998). Parallel micro-assembly. In Workshop on Precision Manipulation at Micro and Nano Scales, IEEE Int. Conf. on Robotics and Automation, 110-135, https://doi.org/10.1177/0278364908097586

Boonrong, P., \& Kaewkamnerdpong, B. (2011). Canonical PSO based nanorobot control for blood vessel repair. World Academy of Science, Engineering and Technology, 58, 511-516.

Boor, V., Overmars, M., \& van der Stappen, A. F. (1999). The Gaussian sampling strategy for probabilistic roadmap planners. In Proc. of IEEE Int. Conf. Robotics and Automation, Detroit, MI. https://doi.org/10.1109/ROBOT.1999.772447

Boulic, R., Mas, R., \& Thalmann, D. (1997). Complex character positioning based on a compatible flow model of multiple supports. IEEE Transactions on Visualization and Computer Graphics, 245-261. https://doi.org/10.1109/2945.620491

Cavalcanti, A., Shirinzadeh, B., \& Kretly, L. C. (2008). Medical Nano-robotics for Diabetes Control. Nanomedicine: Nanotechnology, Biology and Medicine, Elsevier, 4(2), 127-138. https://doi.org/10.1016/j.nano.2008.03.001

Cavalcanti, A., Shirinzadeh, B., Fukuda, T., \& Ikeda, S. (2009). Nano-robot for Brain Aneurysm. International Journal of Robotics Research, Sage, 28(4), 558-570.

Cavalcanti, A., Shirinzadeh, B., Fukuda, T., \& Seiichi, I. (2007). Hardware Architecture for Nano-robot Application in Cerebral Aneurysm. IEEE Nano Int'l Conf. on Nanotechnology, Hong Kong, China, 237-242.

Cavalcanti, A., Shirinzadeh, B., Hogg, T., \& Kretly, L. C. (2006). CMOS-based Nanorobot to Combat Cancer. Australian Workshop on Fluid Mechanics, A Complex Dynamical System, Melbourne, Australia, December 2006.

Cavalcanti, A., Shirinzadeh, B., Murphy, D., \& Smith, J. A. (2007). Nanorobots for Laparoscopic Cancer Surgery. IEEE ICIS Int'l Conf. on Computer and Information Science, Melbourne, Australia, 743-738. https://doi.org/10.1109/ICIS.2007.138

Chenney, S. (1996). Sensing for autonomous agents in virtual environments http://http.cs.berkeley.edu/ schenney/autonomous/sensing.html, 1996.

Ching, W., \& Badler, N. (1992). Fast motion planning for anthropometric figures with many degrees of freedom. In Proc. IEEE Int. Conf. on Robotics and Automation, 2340-2345. https://doi.org/10.1109/ROBOT.1992.220113

Dilts, D. M., Boy, N. P., \& Whoirms, H. H. (1991). The evolution of control architectures for automated manufacturing systems. In Journal of Manufacturing Systems, 10. https://doi.org/10.1016/0278-6125(91)90049-8

Douglas, S. M., Bachelet, I., \& Church, G. M. (2012). A Logic-Gated Nanorobot for Targeted Transport of Molecular Payloads. Science, 335(6070), 831-834. https://doi.org/10.1126/science.1214081

Drexler, K. E. (1981). Molecular Engineering: an Approach to the Development of General Capabilities for Molecular Manipulation. Proc. Natl. Acad. Sci., USA, 78(9), 5275- 5278. https://doi.org/10.1073/pnas.78.9.5275

Farr, R., Choi, D. S., \& Lee, S. W. (2014). Phage-Based Nanomaterials for Biomedical Applications. Acta Biomaterialia, 10(4), 1741-50. https://doi.org/10.1016/j.actbio.2013.06.037

Fatemeh, M., \& Habib, N. K. (2007). Microsurgery design using several degrees of freedom micromanalytic. Comprehensive site of Medical Engineering.

Feynman, R. P. (1960). There's Plenty of Room at the Bottom. Caltech's Engineering and Science, Feb., 22-36. 
Hsu, D., Kavraki, L. E., Latombe, J. C., Motwani, R., \& Sorkin, S. (1998). On finding narrow passages with probabilistic roadmap planners. In P.K. Agarwal, L.E.Kavraki, and M.T. Mason, editors, Robotics: The Algorithms Perspective, Workshop on Algorithmic Foundations of Robotics, A. K. Peters, Natick, MA, 141-153.

Hsu, D., Latombe, J. C., \& Motwani, R. (1997). Path planning in expansive configuration spaces. Int. J. of computational Geometry and Applications, 9(4-5), 495-512. https://doi.org/10.1109/ROBOT.1997.619371

Jaiswal, A., Thakar, H., Atanukumar, B., Krunali, T., \& Meshram, D. B. (2013). Nanotechnology revolution: respirocytes and its application in life sciences. Innovative Journal of Life Sciences, 1(1), 8-13.

Koga, Y., Kondo, K., Kuffner, J., \& Latombe, J. C. (1994). Planning motions intentions. In Proc. SIGGRAPH', 94, 395-408. https://doi.org/10.1145/192161.192266

Kuffner, J. J. Jr. (1998). Goal-directed navigation for animated characters using real-time path planning and control. In Proc. of CAPTECH'98 Workshop on Modeling and Motion Capture Techniques for Virtual Environments. Springer-Verlag.

Mokhoff, N. (2003). Education Overhaul Urged for Nanotech Revolution. EE Times, February 2003, http://www.theworkcircuit.com/news/OEG20030206S0026

Requicha, A. A. G. (2003). Nano-robots, NEMS and Nano-assembly. IEEE ICRA International Conference on Robotics and Automation, 91(11), 1922-1933.

Scientific American (2002). O Brasil na era da nano-tecnologia. Scientific American Brasil, 26 September 2002. http://www2.uol.com.br/sciam/brasil.htm

Sivasankar, M, \& Durairaj, R. B. (2012). Brief Review on Nano Robots in Bio Medical Applications. J. Adv. Robot Automat 1(101). https://doi.org/10.4172/2168-9695.1000101

Watson, B., Friend, J., \& Yeo, L. (2009). Piezoelectric ultrasonic resonant motor with stator diameter less than $250 \mu \mathrm{m}$ : the Proteus motor. Journal of Micromechanics and Micro engineering, 19(2), 022001 (5pp). https://doi.org/10.1088/0960-1317/19/2/022001

\section{Copyrights}

Copyright for this article is retained by the author(s), with first publication rights granted to the journal.

This is an open-access article distributed under the terms and conditions of the Creative Commons Attribution license which permits unrestricted use, distribution, and reproduction in any medium, provided the original work is properly cited. 